\title{
A STUDY OF THE EXTERNAL FINGER FIXATOR TO CORRECT FLEXION DEFORMITY
}

\author{
H. S Abd Rahman ${ }^{1}$, N. A. Abu Osman ${ }^{1}$, S. Yahud ${ }^{1}$, T. S. Tunku Yahya ${ }^{2}$ and Ng Eng Seng ${ }^{2}$ \\ ${ }^{1}$ Department of Biomedical Engineering, Faculty of Engineering, University of Malaya, \\ ${ }^{2}$ Department of Orthopedic, Faculty of Medicine, University of Malaya \\ email: herman.shah@um.edu.my, azuan@um.edu.my
}

\begin{abstract}
INTRODUCTION
Severe fixed flexion deformities at the fingers are common injuries that can lead to pain and stiffness when treated improperly. The damage varies from a sprain of the collateral ligaments to an all-round comminuted fracture-dislocation. The flexion deformities occur because of a variety of issues including fractures (broken bones), burns, tissue expansion, facial contracture and finger joint dislocation [1]. With conventional splints it is difficult to apply an effective torque without causing pain and inflammation due to pressure on the skin. The objective of the study is to develop a new design for external finger fixator to correct severe fixed flexion deformity. Siow et al. (1999) conducted a clinical trial on three patients by using mini external fixator where each of them had severe fixed flexion of deformity. The device was applied up to 44 days and post-operative observation showed that the effected finger can extend up to $75 \%$ from the initial range of motion [2]. The use of this external fixator for correction of rigid soft tissue deformities of the hand has proved that it is not only feasible, but works well.
\end{abstract}

\section{METHODS}

The new external finger fixator (AHS e-FingerFix) was composed of 3 semi-circular rings constructed with 2 long threaded rods used to flex or extend the fixator by turning the knobs. Each of semi-circular rings is made of 2 h-shape. The methodology of this study can be divided into 3 main steps. The first step is to design new external finger fixator with some modifications to improve the previous finger fixator. The design was made by using ProEngineer ${ }^{\circledR}$ version 2.0. During the design period, Finite Element Analysis (FEA) was applied to measure the fixator stiffness and fracture gap motion to be obtained on variety of loading conditions. This step will include the analytical and finite element models to enable the stiffness of a given geometry of external fixator to be predicted accurately. The next step is to apply the device to a number of patients; to see whether the new fixator can withstand to a severe fixed flexion deformity. The stresses and strains that prevail over scar tissue of various types and thicknesses were measured. Figure 1 show that the device was also applied to a cadaver to observe the flexion and extension of the finger.

\section{RESULTS AND DISCUSSION}

The idea to turn out into a new design was driven by the problems which arise from the previous finger fixator [2]. All parts of the new design for the external finger fixator can easily be taking to pieces so that the surgeon or hand therapist, without difficulty can dismantle the part that are not helpful because of the effected area (stiff joint), congenital deformities, fractures and arthrodesis had been cured.

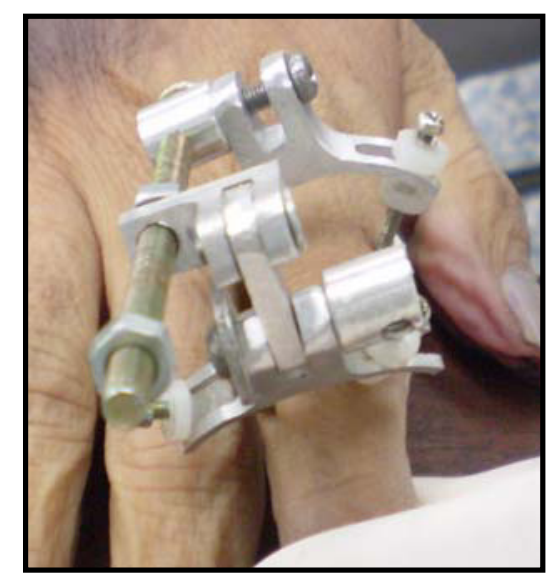

Figure 1: The AHS e-FingerFix was attached to a cadaver by a surgeon

The angle of the pin that attached to each phalanx also inherits modification where the angle of the pin is not horizontal with sagittal plane but it can be ranging from $5^{\circ}$ to $60^{\circ}$ (depends on the size of the phalanx bone and to the surgeon itself). This will be a huge advantage to the surgeon where it will be more flexible to choose the correct angle of drilling the threaded pin. This is because of the half moon shape of the phalanges bony architecture. Some plates will be attached by hinges and this will allow movement from 0 to 110 degree to allow for the angle of deformity and its correction (Figure 2).

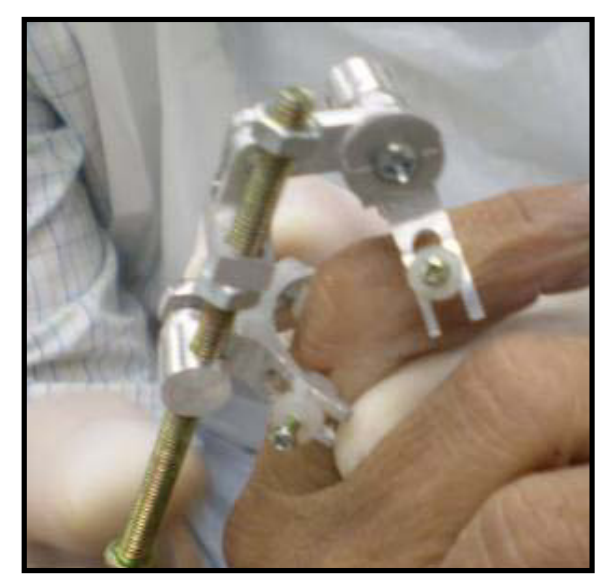

Figure 2: AHS e-FingerFix provide an extension up to $85 \%$ from initial range of motion

\section{REFERENCES}

1. Citron N. and Messina JC. JBJS Br; 80(1), 126-129, 1998

2. Siow Y.S., et al. Hand Surgery, 4(2): 167-174, 1999 